\title{
Nanostructured Material Fabrication for Energy Conversion
}

\author{
G. Ferrara ${ }^{a}$, R. Inguanta ${ }^{a}$, F. Vergottini, ${ }^{a}$ S. Piazza ${ }^{a}$, C. Sunseri ${ }^{a}$ \\ ${ }^{a}$ Department of Chemical Engineering, Palermo University, Palermo, 90128, Italy
}

The electrochemical deposition is a suitable via to fabricate nanostructured materials for energy conversion, and for other purposes. This paper deals with the electrochemical synthesis of nanostructured alloys and ruthenium oxide, which can be used in Li-ion batteries and polymer electrolyte membrane electrolyzers.

\section{Introduction}

In recent years, growing interest has been addressed to development of energy storage systems for power supply, mainly, of electronic portable devices. Recent improvements in the performances of the batteries has driven the development of electric or hybrid vehicles with growing request of high power batteries having enhanced performances. In this context, lithium-ion batteries (LIBs) are playing a fundamental role. Another technologically exciting way to store energy is hydrogen production by water electrolysis coupled to renewable energy sources. In this case, hydrogen can be used either for supplying fuel cells (cold combustion) or for usual hot combustion. For development of both energy storing ways, extensive research activities are requested in order to find materials with enhanced electrochemical performances even if this is only the first challenge to be faced.

About LIBs, many efforts are devoted to the use of tin as anodic material owing to its high theoretical specific capacity of $994 \mathrm{mAh} \mathrm{g}^{-1}$, related to $\mathrm{Li}_{4.4} \mathrm{Sn}$ formation. This value is significantly higher than that of currently used carbonaceous materials ( $\left.372 \mathrm{mAh} \mathrm{g}^{-1}\right)$. The main drawback, limiting the use of tin as anodic active material, is due to its fast decay under charge/discharge cycles. As lithium is intercalated (charging step), tin specific volume increases of about 300\% with consequent onset of high mechanical stresses, which relax during the following discharge step. Repeated cycles lead to a rapid failure of the battery owing to the crumbling of the anode. This drawback has been overcome by Sony with the synthesis of an amorphous SnCo alloy (1) where cobalt is electrochemical inactive but buffers mechanical stresses. The mechanical decay of tin based anodes can be further inhibited by reducing the size of reacting particles up to nano-scale (2). In addition, the use of nanostructured materials in LIBs improves the effective capacity of the battery and greatly reduces the charging time (3-4). For these reasons, we focused our attention on these kind of materials. In the present work, we present some results about the synthesis of one-dimensional nanostructured tin based alloys, by electrodeposition in template. In particular, polycarbonate membranes (PCM) has been found more suitable than anodic alumina membrane (AAM) as template, because it can be easily removed by etching in organic solvent without any damage of the electrodeposited alloy. In the frame of a more complete investigation on the synthesis of materials for energy storage, the attention has been also devoted to electrodes for Polymer Electrolyte Membrane Electrolyzer (PEMEL). Here, we will present some 
preliminary results on electrochemical preparation of both PdCo alloys, to be used as cathode for hydrogen production in place of the more expensive Pt-based electrocatalyst (5), and $\mathrm{RuO}_{2}$ as base material for high efficient anodes for water oxidation process [6]. PdCo alloys were directly deposited on carbon paper acting as current collector/gas diffusion layer of a PEMEL cathode, while $\mathrm{RuO}_{2}$ was also deposited in the form of nanotubes inside the channels of an AAM acting as template. All materials were characterized by X-Ray Diffractometry, (XRD) and Scanning Electron Microscopy (SEM). In addition, electrodeposited $\mathrm{RuO}_{2}$ was also characterized by Raman Spectroscopy. The cathodic behaviour of PdCo alloys was also investigated.

\section{Tin-based nanostructured alloys}

Nanostructured $\mathrm{SnCo}$ and $\mathrm{SnCu}$ alloys were fabricated by electrochemical deposition in template. The former is the tin alloy that gives the best performance in terms of cycle number (7), whilst the last is preferable from both economic and environmental point of view because $\mathrm{SnCu}$ is cheaper and more environmental friendly than $\mathrm{SnCo}$, even if the amount of Co alloyed to Sn is very low. The addition of Co is necessary in order to improve the dimensional stability of the anode, and its amount depends on the morphology of the active material. In particular, its size reduction favors the mechanical stability of tin because the strained region is less large, consequently in the presence of nanostructured morphology the amount of the Co to be added decreases with the advantage of a higher specific capacity.

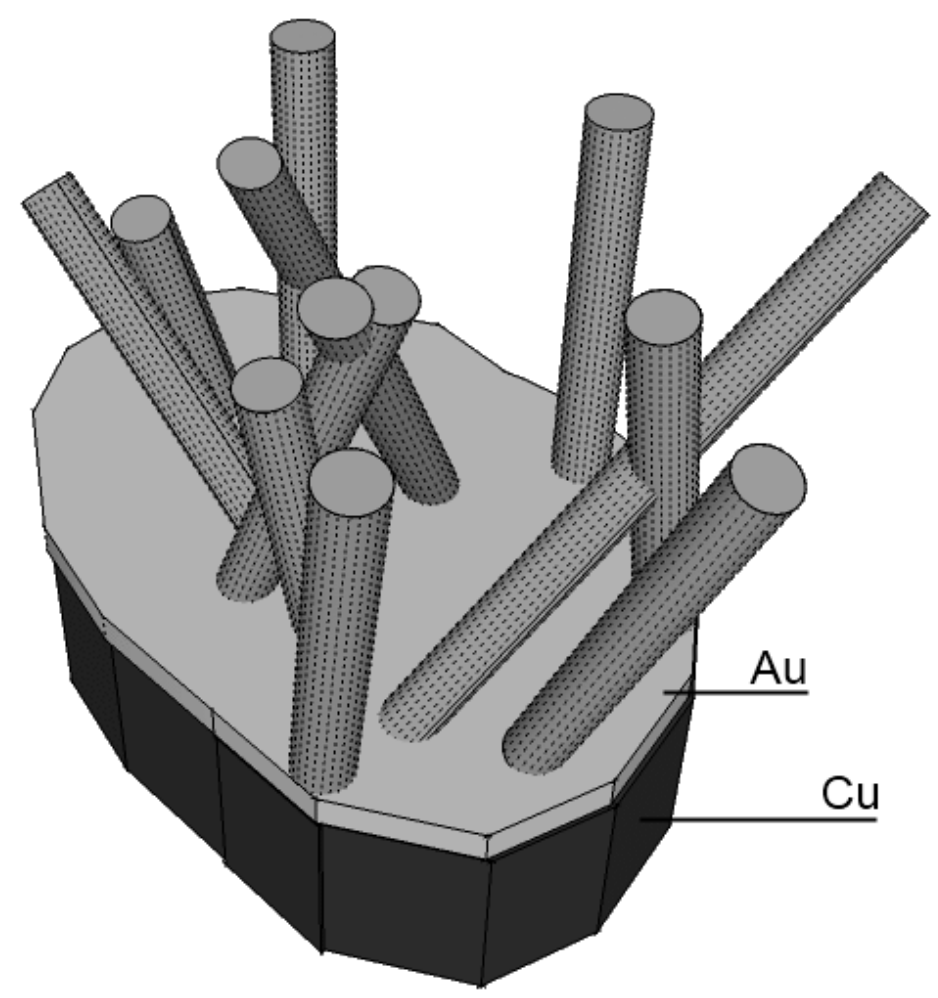

Figure 1. Scheme of the morphological structure of tin-based nanowires connected to the current collector after dissolution of the template 
Track-etched polycarbonate membranes (PCMs) by Whatman (Nuclepore ${ }^{\mathrm{TM}}$ ) were used as template for synthesizing the nanostructured alloys. These membranes are characterized by interconnected cylindrical pores with a diameter of about $200 \mathrm{~nm}$. Prior to conducting electrodeposition, a gold film, about $30 \mathrm{~nm}$ thick, was sputtered on one side of the membrane. Then, copper current collector was electrodeposited on the gold film, followed by electrodeposition of SnM nanowires (NWs) inside the pores of the PCM. The use of a home-made holder, specifically designed, allowed to conduct both electrodepositions in a two steps process, avoiding the dismount of the electrode after the first electrodeposition. By this procedure, NWs were firmly connected to copper current collector, ensuring a good electric contact, without the use of conductive binder that are currently used for performing low resistance contact between active materials and current collector. Electrodepositions were conducted at constant potential, referred to Saturated Calomel Electrode (SCE), in a three electrode cell with platinum wire as counter electrode. After deposition of current collector and alloy NWs, template was dissolved in $\mathrm{CHCl}_{3}$ and the morphological structure sketched in Fig. 1 was obtained.

SEM image of Fig. 2 shows typical SnCo morphology NWs fabricated by electrodeposition in template. This image is also rapresentative of $\mathrm{SnCu}$ alloy because the morphology of the NWs mainly depends on the nature of the template. Fig. 2 shows that NWs are interconnected, but there is sufficient free space around every NW that may guarantee good performances as anode of Li-ion battery. In this application, electrode is subject to large volume changes due to intercalation/deintercalation of $\mathrm{Li}$, that, in the case of pure tin, can reach a value of $300 \%$. Consequently, free space is fundamental to accommodate large change volumes avoiding damage of the NWs. Another advantage related to the distance among NWs, is the complete wetting of the surface by the electrolytic solution leading to a high utilization degree of the electrode. Low free space between NWs, like in the case of NWs grown inside anodic alumina membrane, can more or less hindering the penetration of the solution leading to a low utilization degree of active material in addition to possible damage due to volume expansion.

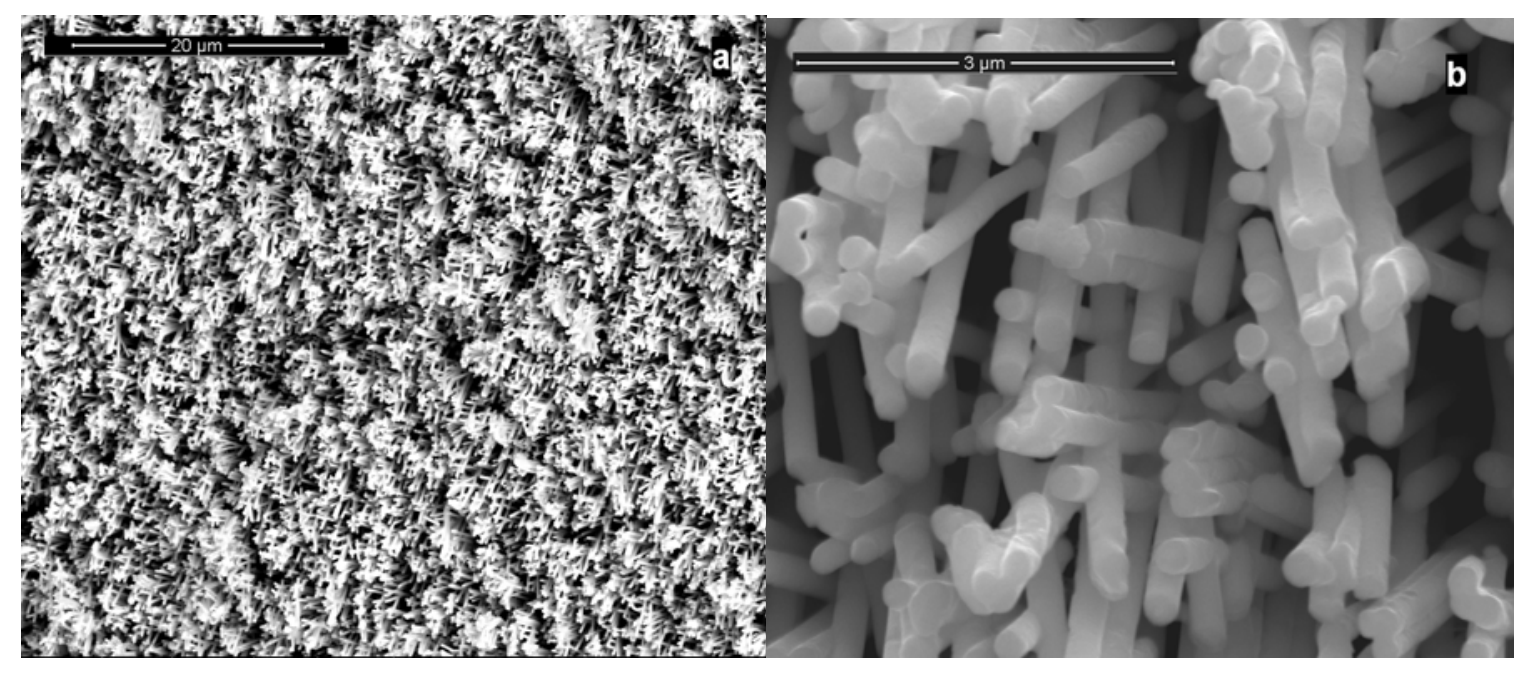

Figure 2. SEM images of SnCo nanowires fabricated by electrodeposition inside the pores of a track-etched polycarbonate membrane, after template dissolution. 
$\mathrm{XRD}$ analysis of tin alloys are reported in Fig. 3. Both $\mathrm{SnCo}$ and $\mathrm{SnCu}$ diffractograms always reveal the presence of peaks due to the copper current collector (ICDD-card \#040836). Fig. 3a shows that the SnCo alloy is amorphous, with a peak which could be attributed to the (200) Sn crystallographic plane (ICDD-card \#04-0673). The peak at $2 \theta=$ 32.92 might be attributed to an unknown tin-cobalt phase, according to (8). Besides, Gómez et al. (9) have electrodeposited a crystalline tin cobalt alloy and have proposed a new SnCo tetragonal phase with a main peak at $2 \theta=32.77$, very close to the second unknown peak of fig. 3a. However, taking into account that, except for copper peaks, the other ones were broad and weak, it can be concluded that low crystalline SnCo NWs were electrodeposited.

$\mathrm{SnCu} \mathrm{XRD}$ pattern of Fig. 3b shows the presence of two crystalline phases that were attributed to $\mathrm{Sn}$ and $\mathrm{Cu}_{6} \mathrm{Sn}_{5}$ according to ICDD-card \#45-1488. The grain sizes of these phases were evaluated by Scherrer's equation (10) and values of $22 \mathrm{~nm}$ and $24 \mathrm{~nm}$ were found for $\mathrm{Sn}$ and $\mathrm{Cu}_{6} \mathrm{Sn}_{5}$, respectively. Further work is in progress in order to fabricate $\mathrm{SnCu}$ alloy with a lower crystalline degree, since it is reported in the literature that more or less amorphous alloy present better performance than crystalline form. Electrochemical characterization of SnCo NWs as anodic material for LIBs (not showed here) are in progress and preliminary results were reported elsewhere (11).

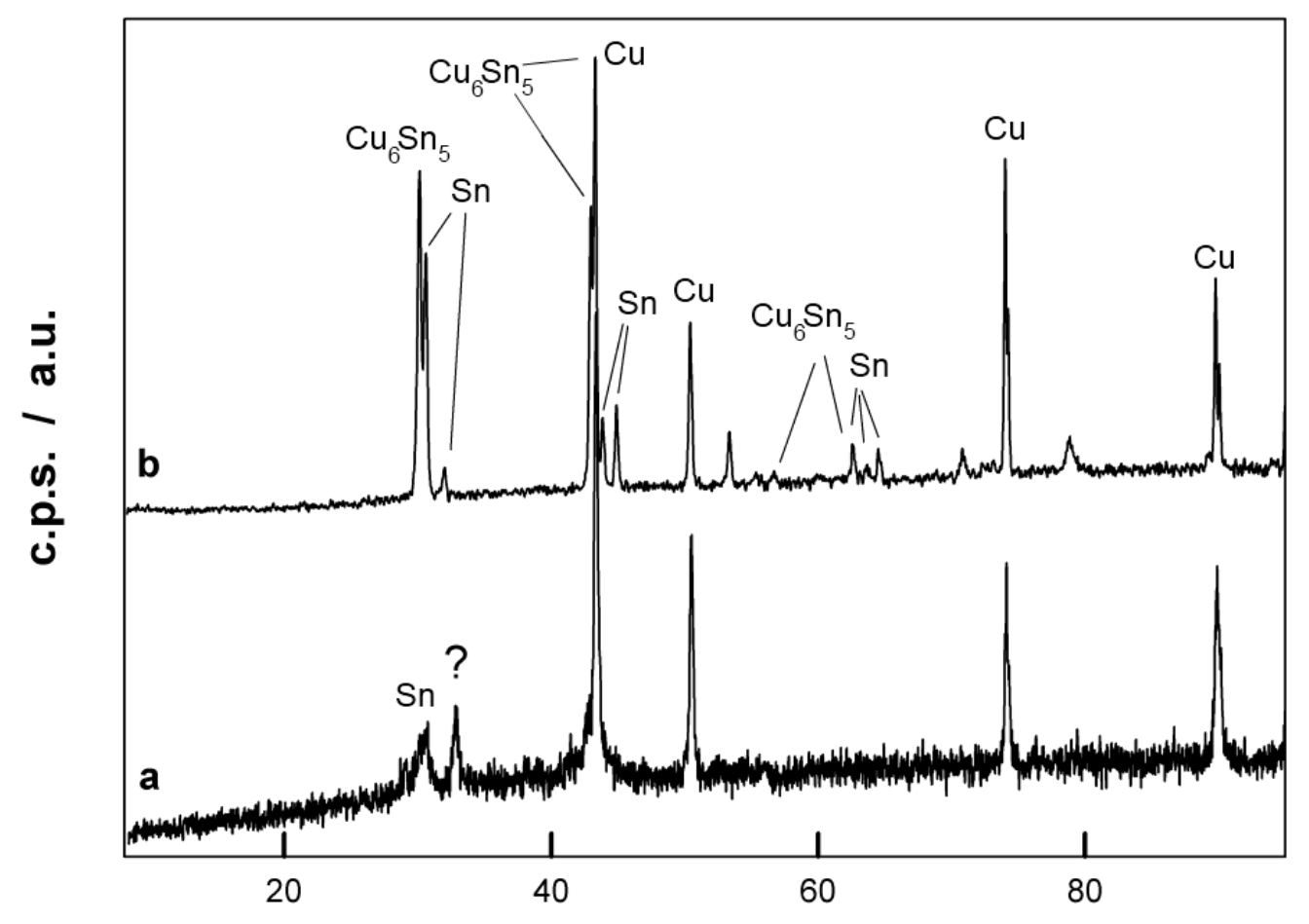

$2 \theta / \mathrm{deg}$

Figure 3. XRD of $\mathrm{SnCo}(\mathrm{a})$ and $\mathrm{SnCu}(\mathrm{b})$ nanowires after template removal. 


\section{Materials for electrolyzers of PEM type}

A two-electrode cell, specifically designed, was used in order to reduce electrolyte volume. For this aim, we have used a tube glass with a screw-thread (SVL® 15) that was modified in order to get a pipe-like support [12]. This choice was took because of our willing of synthesizing technologically relevant materials at lower price, even considering that reagents are very valuable. Two different types of support (carbon paper (CP) and AAM) were used as working electrode with a area exposed to the solution of $0.5 \mathrm{~cm}^{2}$. The cathodic galvanostatic electrodeposition of $\mathrm{RuO}_{2}$ and $\mathrm{PdCo}$ alloy was carried out in aqueous solution at room temperature.

\section{Binary PdCo alloys.}

PdCo depositions were conducted at constant current density ranging between 1.56 and $156 \mathrm{~mA} \mathrm{~cm}^{-2}$, for times varying from 60 to 1800 seconds, using very dilute solution of $\mathrm{Pd}(\mathrm{II})$ and $\mathrm{Co}(\mathrm{II}) . \mathrm{Pd} / \mathrm{Co}$ ratios were changed. Figure 4 shows that carbon paper fibers were covered with active material, whose morphology changed from nanoparticles (Fig. 4a), uniformly distributed on CP fibers with a mean dimension of $20 \mathrm{~nm}$, to continuous thin films (Fig. 4b) that fully covered fibers. We have found that $900 \mathrm{sec}$ is the optimal deposition time because for longer time than $900 \mathrm{sec}$, we found that PdCo deposit was tending to detaching from the fibers.

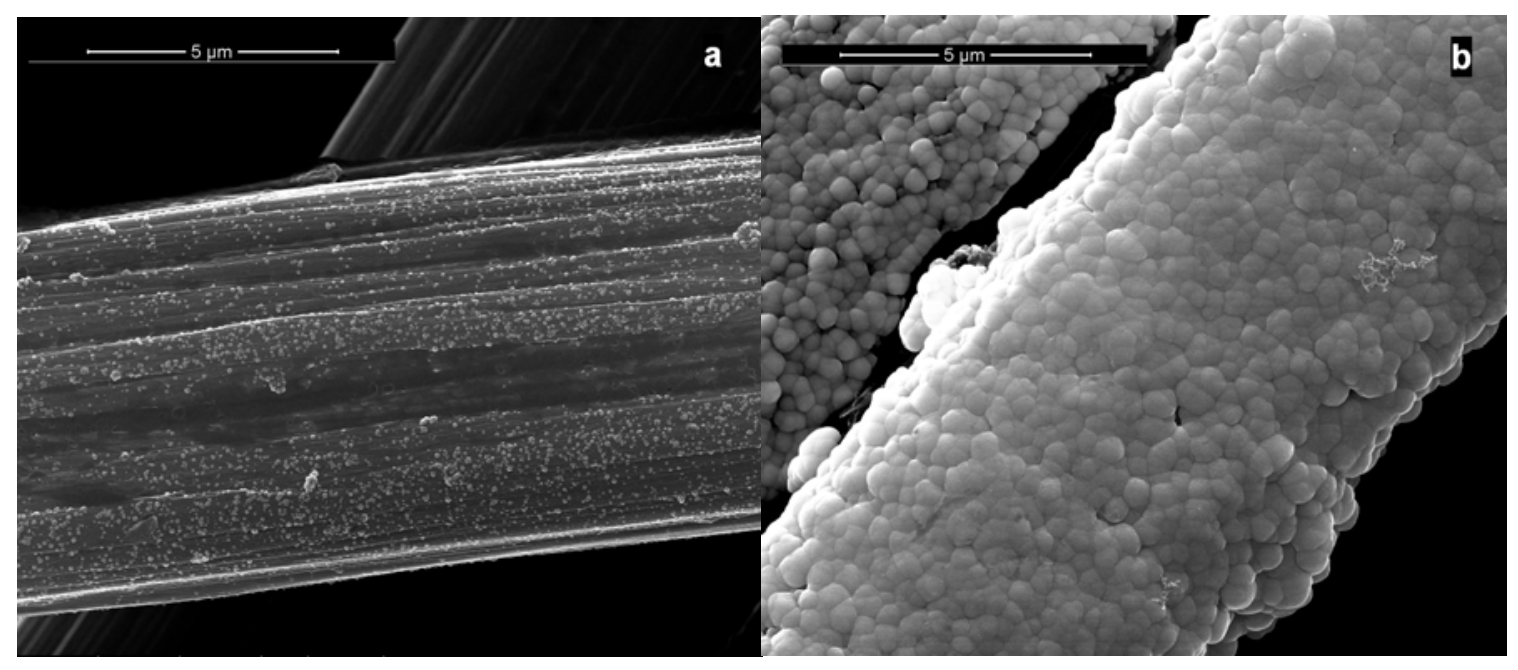

Figure 4. Morphology of PdCo alloy obtained at different electrodeposition conditions: (a) $60 \mathrm{sec}, 15.6 \mathrm{~mA} \mathrm{~cm}^{-2}$; (b) $900 \mathrm{sec}, 15.6 \mathrm{~mA} \mathrm{~cm}^{-2}$. 


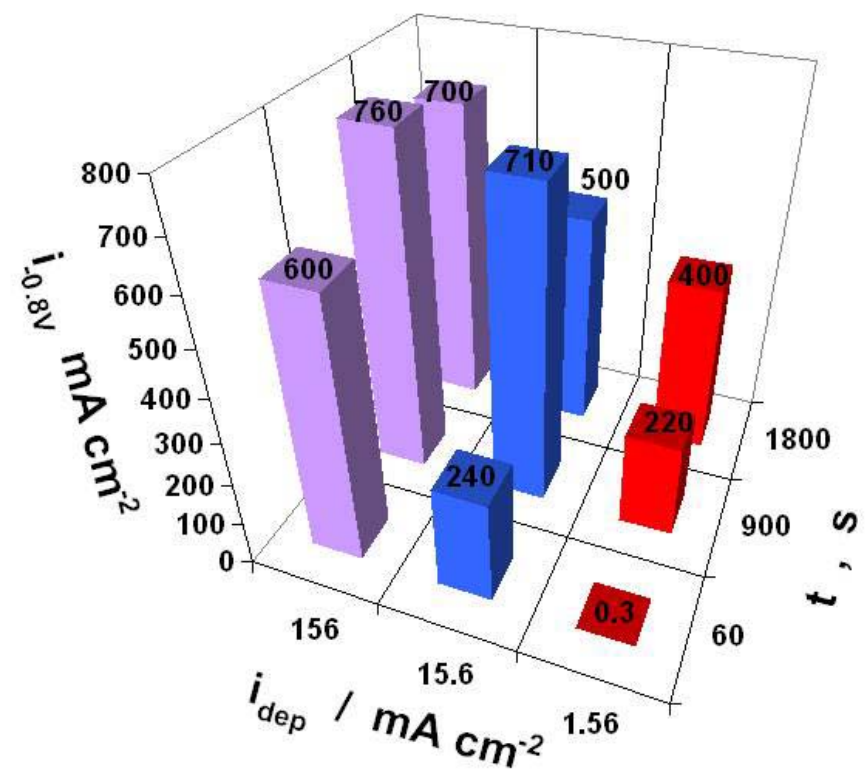

Figure 5. Current density at $-0.8 \mathrm{~V}(\mathrm{NHE})$ for hydrogen evolution reaction on $\mathrm{PdCo}$ electrodes obtained at different electrodeposition conditions.

The electrochemical behavior of deposits has been studied by cyclo-voltammetry and chrono-potentiometric tests, performed in $2 \mathrm{M} \mathrm{H}_{2} \mathrm{SO}_{4}$ solution. The performance of these deposits changed with alloy composition and morphology. As shown in Figure 5, PdCo alloys obtained at higher current density exhibit higher hydrogen evolution rate.

$\underline{\mathrm{RuO}_{2}}$.

Fig. 6 and 7 show FEG-SEM pictures of $\mathrm{RuO}_{2}$ film (Fig. 6a-b) and NTs after total template dissolution (Fig. 7a-b). Fig. 6 shows that all fibers of CP were covered with $\mathrm{RuO}_{2}$ deposit that, in many areas, led to the formation of a continuous and compact film. $\mathrm{RuO}_{2}$ NTs are clearly visible in Fig. 7: their population density is of the order of $10^{13}$ NTs $\mathrm{m}^{-2}$. The mean external diameter is $210 \mathrm{~nm}$ and the tubular structure extends throughout the length of NTs, although mean wall thickness changes from $65 \mathrm{~nm}$ at the bottom to 40 $\mathrm{nm}$ at the top. 


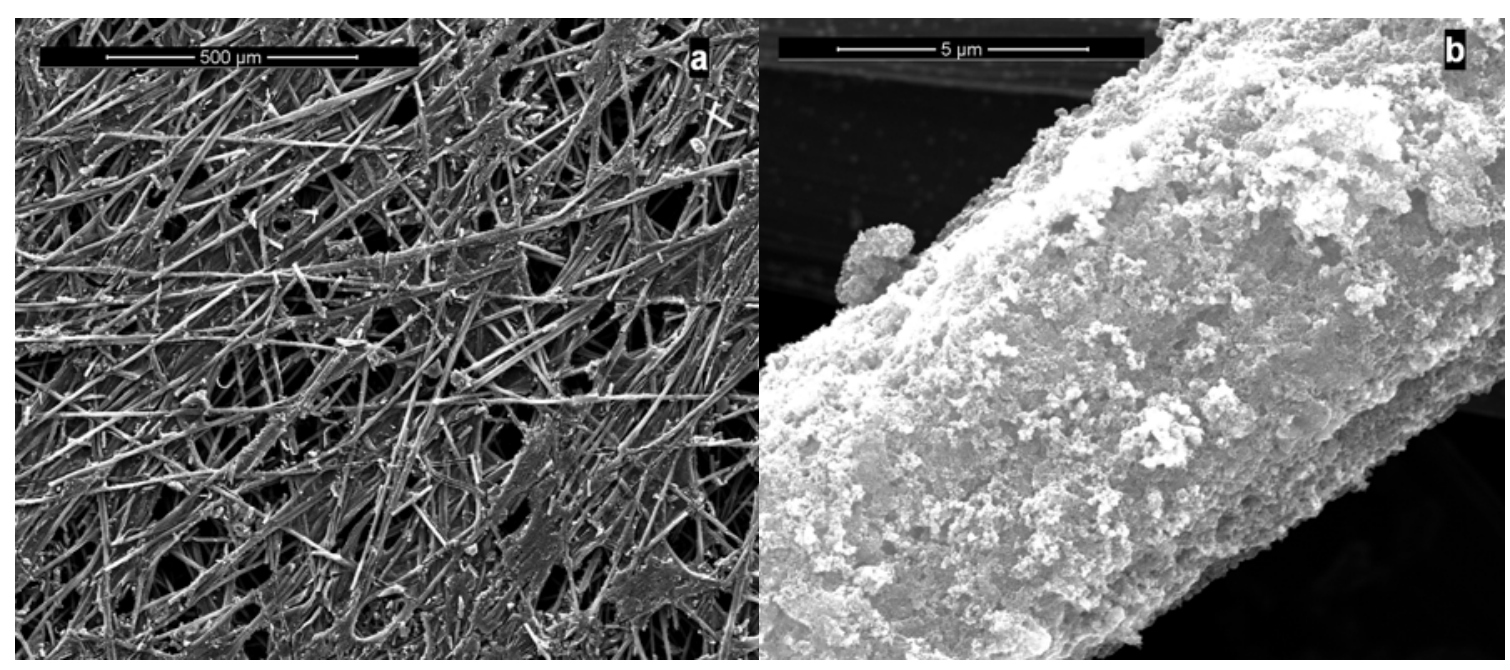

Figure 6. SEM images of $\mathrm{RuO}_{2}$ deposits grown onto carbon paper fibers.

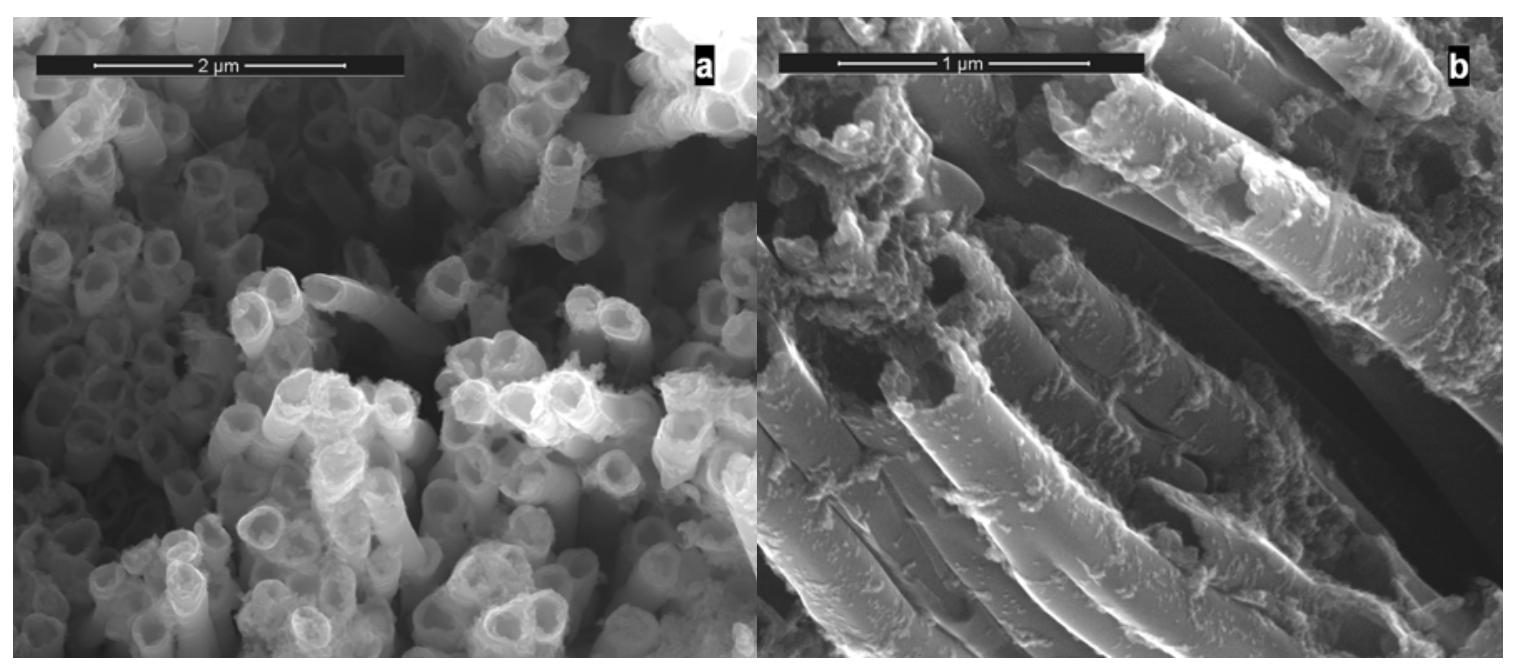

Figure 7. SEM images of $\mathrm{RuO}_{2}$ deposits grown in anodic alumina membrane.

In order to identify the deposit obtained by electrodeposition, XRD, EDS, and Raman analyses were conducted on the as-prepared samples. In particular, on the basis of comparison of XRD patterns performed on substrate before and after the electrodeposition processes, we can conclude that the deposit consists of an amorphous phase, in agreement with the result obtained by Fang et al (13). 


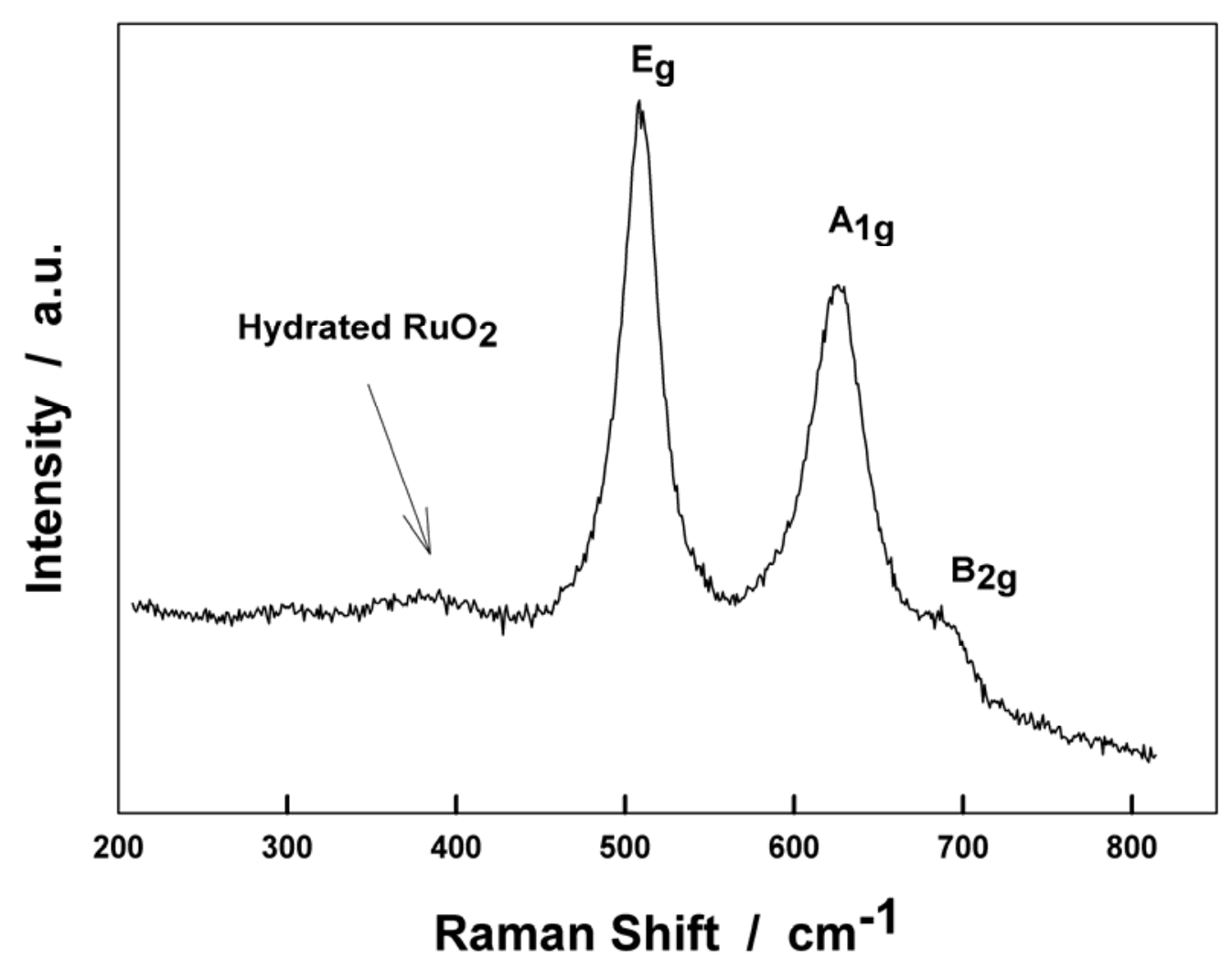

Figure 8. Raman spectrum of RuO2 NTs obtained in AAM at $5 \mathrm{~mA} \mathrm{~cm}-2$ for $3 \mathrm{~h}$.

In the case on nanotubes, Raman spectrum was reported in Fig. 8. The presence of three main Raman peaks can be observed, that were assigned to the $\operatorname{Eg}\left(508.6 \mathrm{~cm}^{-1}\right), \mathrm{A}_{1 \mathrm{~g}}$ $\left(625.7 \mathrm{~cm}^{-1}\right)$ and $\mathrm{B}_{2 \mathrm{~g}}\left(692.7 \mathrm{~cm}^{-1}\right)$ vibration modes of $\mathrm{RuO}_{6}$ structural unit of $\mathrm{RuO}_{2}$ in the tetragonal form. These results have been obtained identically by Chang et al. in the case of hydrothermal synthesis of $\mathrm{RuO}_{2}$ nanoparticles [14]. Furthermore, it can be observed a weak and broad peak at $390 \mathrm{~cm}^{-1}$, which has not yet been identified. Probably, according to the spectra reported by Lee et al. [15], can be assigned to the hydrated form of ruthenium oxide. It is important to highlight that, for the single crystal $\mathrm{RuO}_{2}$, the three Raman modes are located at about 528, 646, and $716 \mathrm{~cm}^{-1}$ [16]. In our case, all the three peaks show a red-shift of about $20 \mathrm{~cm}^{-1}$ that can be attributed to the nanoscale nature of as-deposited $\mathrm{RuO}_{2}$ as reported by several authors [14-16]. Thus, on the basis of these results we can conclude that the deposit that we have obtained inside the channels of alumina template is amorphous hydrated $\mathrm{RuO}_{2}$.

\section{Conclusions}

A simple, cheap and easily scalable procedure was proposed for synthesizing nanostructured tin alloys which may be used for fabrication of high capacity anodes for lithium-ion batteries. Crystalline structure and morphology of NWs, deposited inside the channels of polycarbonate membrane, can be controlled by adjusting bath composition and electrode polarization. Interconnected nanostructures of binary tin-based alloys were electrodeposited with different crystalline structure. SnCo alloys showed low crystallinity 
degree, while $\mathrm{SnCu}$ was synthesized with a crystalline structure. In view of possible application of these alloys as anodes of Li-ion battery, the free space among NWs should allow to accommodate the large volume changes due to $\mathrm{Li}$ intercalation/deintercalation and to enhance the utilization of the active material.In addition, using a specifically designed electrochemical cell, we have fabricated $\mathrm{RuO}_{2}$ and PdCo alloys in a single-step by galvanostatic deposition on two different substrates. This cell allows to reduce consumption of electrolyte containing precious metals and hence fabrication cost. $\mathrm{RuO}_{2}$ film and NTs were obtained with a nano-crystalline structure as confirmed by both XRD and Raman analyses. SEM investigations of NTs showed an uniform average external diameter with a wall thickness that changed throughout length. PdCo alloys were obtained with different composition and morphology in dependence on electrochemical parameters. These electrodes, tested for hydrogen evolution reaction, showed different performance. Deposit obtained at higher current density exhibit higher hydrogen evolution rate.

\section{Aknowledgments}

This work was financially supported by Università di Palermo.

\section{References}

1. http://www.sony.net/SonyInfo/News/Press/200502/05-006E/

2. C. Jiang, E. Hosono, H. Zhou, Nanotoday, 1, 28 (2006).

3. C. K. Chan, H. Peng, G. Liu, K. Mcllwrath, X. F. Zhang, R. A. Huggins and Y. Cui, Nat. Nanotechnol. 3, 31 (2008).

4. R. Deshpande, Y-T. Cheng, M. W. Verbrugge, J. Power Sources, 195, 5081 (2010).

5. O. Savadogo, K. Lee, K. Oishi, S. Mitsushima, N. Kamiya, K-I. Ota, Electrochem. Comm., 6, 105 (2004).

6. S. Song, H. Zhang, X. Ma, Z. Shao, R. Baker, B. Yi, Int. J. Hydrogen Energy, 33, 4955 (2008).

7. A. D. W. Todd, R. E. Mar, J. R. Dhan, J. Electrochem. Soc., 153, A1998 (2006).

8. J. R. Dahn, R. E. Mar, A. Abouzeid, J. Electrochem. Soc., 153, A361 (2006).

9. E. Gómez, E. Guaus, J. Torrent, X. Alcobe, E. Vallés, J. Appl. Electrochem., 31, 349 (2001).

10. A. R. West, Solid State Chemistry and its Applications, Wiley, Chichester, p. 173 (1985)

11. G. Ferrara, L. Damen, C. Arbizzani, R. Inguanta, S. Piazza, C. Sunseri, M. Mastragostino, J. Power Sources, 196, 1469 (2011).

12. R. Inguanta, G. Ferrara, P. Livreri, S. Piazza, C. Sunseri, Curr. Nanosci., 7, 1 (2011).

13. W-C. Fang, J-H. Huang, L-C. Chen, Y-L. O. Su, K-H. Chen, J Power Source, 160, 1506 (2006).

14. K-H. Chang, C-C. Hu, C-Y. Chou, Chem. Mater, 19, 2112 (2007).

15. S.H. Lee, P. Liu, H. M. Cheong, C. E. Tracy, S. K. Deb, Solid State Ionics, 165, 217 (2003).

16. S. Y. Mar, C. S. Chen, Y. S. Huang, K. K. Tiong, Appl. Surf. Sci., 90, 497 (1995). 